\title{
Small-scale magnetic flux emergence in a sunspot light bridge ${ }^{\star}$
}

\author{
Rohan E. Louis ${ }^{1,2,3}$, Luis R. Bellot Rubio ${ }^{2}$, Jaime de la Cruz Rodríguez ${ }^{4}$, Héctor Socas-Navarro ${ }^{5,6}$, and Ada Ortiz ${ }^{2}$ \\ ${ }^{1}$ Leibniz-Institut für Astrophysik Potsdam (AIP), 16 An der Sternwarte, 14482 Potsdam, Germany \\ e-mail: rlouis@aip.de \\ 2 Instituto de Astrofísica de Andalucía (CSIC), Apartado de Correos 3004, 18080 Granada, Spain \\ 3 Udaipur Solar Observatory, Physical Research Laboratory, Dewali, Badi Road, Udaipur - 313004 Rajasthan, India \\ 4 Institute for Solar Physics, Dept. of Astronomy, Stockholm University, Albanova University Center, 10691 Stockholm, Sweden \\ 5 Instituto de Astrofísica de Canarias, vía Láctea s/n, 38205 La Laguna, Tenerife, Spain \\ ${ }^{6}$ Departamento de Astrofísica, Universidad de La Laguna, 38205 La Laguna, Tenerife, Spain
}

Received 29 June 2015 / Accepted 18 August 2015

\section{ABSTRACT}

Context. Light bridges are convective intrusions in sunspots that often show enhanced chromospheric activity.

Aims. We seek to determine the nature of flux emergence in a light bridge and the processes related to its evolution in the solar atmosphere.

Methods. We analyse a sequence of high-resolution spectropolarimetric observations of a sunspot taken at the Swedish 1-m Solar Telescope. The data consist of spectral scans of the photospheric Fe I line pair at $630 \mathrm{~nm}$ and the chromospheric Ca II $854.2 \mathrm{~nm}$ line. Bisectors were used to construct Dopplergrams from the Fe I $630.15 \mathrm{~nm}$ measurements. We employed LTE and non-LTE inversions to derive maps of physical parameters in the photosphere and chromosphere, respectively.

Results. We observe the onset of blueshifts of about $2 \mathrm{~km} \mathrm{~s}^{-1}$ near the entrance of a granular light bridge on the limbward side of the spot. The blueshifts lie immediately next to a strongly redshifted patch that appeared six minutes earlier. Both patches can be seen for 25 min until the end of the sequence. The blueshifts coincide with an elongated emerging granule, while the redshifts appear at the end of the granule. In the photosphere, the development of the blueshifts is accompanied by a simultaneous increase in field strength of about $400 \mathrm{G}$. The field inclination increases by some $25^{\circ}$, becoming nearly horizontal. At the position of the redshifts, the magnetic field is equally horizontal but of opposite polarity. An intense brightening is seen in the Ca II filtergrams over the blueshifts and redshifts, about $17 \mathrm{~min}$ after their detection in the photosphere. The brightening is due to emission in the blue wing of the Ca II $854.2 \mathrm{~nm}$ line, close to its knee. Non-LTE inversions reveal that this kind of asymmetric emission is caused by a temperature enhancement of $\sim 700 \mathrm{~K}$ between $-5.0 \leq \log \tau \leq-3.0$ and a blueshift of $3 \mathrm{~km} \mathrm{~s}^{-1}$ at $\log \tau=-2.3$ that decreases to zero at $\log \tau=-6.0$

Conclusions. The photospheric blueshifts and redshifts observed in a granular light bridge seem to be caused by the emergence of a small-scale, flat $\Omega$-loop with highly inclined footpoints of opposite polarity that brings new magnetic field to the surface. The gas motions detected in the two footpoints are reminiscent of a siphon flow. The rising loop is probably confined to the lower atmosphere by the overlying sunspot magnetic field and the interaction between the two flux systems may be responsible for temperature enhancements in the upper photosphere/lower chromosphere. This is the first time that magnetic flux is observed to emerge in the strongly magnetised environment of sunspots, pushed upwards by the convective flows of a granular light bridge.

Key words. sunspots - Sun: photosphere - Sun: chromosphere - techniques: high angular resolution - techniques: polarimetric techniques: imaging spectroscopy

\section{Introduction}

Light bridges (LBs) are bright structures in the dark umbra of sunspots (Muller 1979), which bear an umbral, penumbral, or quiet Sun morphology depending on their lifetimes (Sobotka et al. 1994; Lites et al. 2004; Rimmele 2008; Louis et al. 2012). They are often seen during the formation and decay of sunspots along fissures where individual fragment spots coalesce or split (Garcia de La Rosa 1987). These structures are conceived to be either "field-free" intrusions of plasma in the umbral magnetic field (Parker 1979; Choudhuri 1986; Spruit \& Scharmer 2006) or signatures of magneto-convection (Rimmele 2004).

Many LBs show a central dark lane running parallel to their axis and tiny bright grains or granules (Sobotka et al. 1994; Hirzberger et al. 2002; Berger \& Berdyugina 2003; Lites et al. 2004; Rimmele 2008). The dark lane appears to be the

^ The movie associated to Fig. 2 is available in electronic form at http://www. aanda.org result of a hot upflowing plume braked by the surrounding magnetic field, which forces the plume into a cusp-like shape (Schüssler \& Vögler 2006; Cheung et al. 2010). Upflows of nearly $500 \mathrm{~m} \mathrm{~s}^{-1}$ are sometimes seen along the central dark lane, flanked by less vigorous downflows (Rouppe van der Voort et al. 2010). Giordano et al. (2008) reported weaker upflows of about $70 \mathrm{~m} \mathrm{~s}^{-1}$ along the dark lane and downflows of $150 \mathrm{~m} \mathrm{~s}^{-1}$ sideways.

The magnetic field in LBs is weak and inclined (Leka 1997; Jurčák et al. 2006; Katsukawa et al. 2007), possibly because of the magnetic canopy formed by the adjacent umbral field over the LB (Jurčák et al. 2006). This complex and dynamic magnetic configuration is believed to be responsible for a wide range of chromospheric and coronal activity, including surges in $\mathrm{H} \alpha$ (Asai et al. 2001; Guo et al. 2010), C-class flares (Berger \& Berdyugina 2003), coronal jet ejections (Liu 2012), and persistent chromospheric brightenings (Louis et al. 2008, 2014; Shimizu et al. 2009). Some of these brightenings are co-spatial 
with supersonic downflows in the photosphere (Louis et al. 2009). This kind of activity has been attributed to magnetic reconnection (Matthews et al. 2012), although other mechanisms cannot be ruled out (Shimizu 2011).

Our knowledge of the general magnetic structure of LBs is still based on snapshots that reflect different phases of their evolution. Apart from imaging observations, which are routinely carried out (Hirzberger et al. 2002; Katsukawa et al. 2007; Louis et al. 2012), only a handful of spectroscopic or polarimetric time sequences of LBs have been acquired in the past (Schleicher et al. 2003; Rimmele 2008; Rezaei et al. 2012; Sobotka et al. 2013). The evolution of the magnetic and velocity fields on short timescales is dictated by processes that are ultimately responsible for the physical nature and dynamism of LBs. Thus it is important to ascertain how the photospheric magnetic field in LBs influences the overlying chromosphere at the relevant timescales. This kind of study demands high-resolution spectroscopy and polarimetry in the two atmospheric layers with good temporal cadence.

Here we report on the emergence of small-scale magnetic flux in a granular LB using a time sequence of spectropolarimetric observations taken at the Swedish 1-m Solar Telescope. The response of the chromosphere to this event is seen as strong brightenings at the location of the emerging flux nearly $17 \mathrm{~min}$ after the magnetic field appears on the photosphere. We describe the observed Stokes profiles and invert them to investigate the properties of the emerging flux, its evolution, and its influence on the chromosphere.

\section{Observations and data processing}

On 2009 July 5, the leading sunspot of AR 11024 was observed with the CRisp Imaging SpectroPolarimeter (CRISP; Scharmer et al. 2008) at the Swedish 1-m Solar Telescope (Scharmer et al. 2003a). CRISP is a dual Fabry-Pérot system capable of performing quasi-simultaneous measurements in several spectral lines with full Stokes polarimetry. On that particular day, we acquired spectropolarimetric scans of the Fe I lines at 630.15 and $630.25 \mathrm{~nm}$ and the Ca II line at $854.2 \mathrm{~nm}$.

Each of the two Fe lines was scanned at 15 wavelength positions in steps of $4.8 \mathrm{pm}$, covering a spectral window of -33.6 to $+33.6 \mathrm{pm}$ from line centre. In addition, we observed a continuum point at $630.320 \mathrm{~nm}$. Ca II $854.2 \mathrm{~nm}$ was scanned at 17 wavelength positions from -80 to $+80 \mathrm{pm}$ in steps of $10 \mathrm{pm}$, plus a continuum point at $+0.24 \mathrm{~nm}$. Polarimetry was carried out by modulating the incoming light with liquid crystal variable retarders. At each wavelength position, nine exposures per modulation state were taken, resulting in a total of 36 exposures. The time needed to scan the two Fe lines and the continuum point was $33 \mathrm{~s}$. Changing the prefilter took another $5 \mathrm{~s}$, followed by 19 s to scan the Ca line.

The observations were reduced using the CRISP data pipeline (de la Cruz Rodríguez et al. 2015b) with the telescope model of Selbing (2010). High spatial resolution was achieved by means of the adaptive optics system of the SST (Scharmer et al. 2003b) and the multi-object multi-frame blind deconvolution technique (van Noort et al. 2005).

The seeing conditions were good and allowed us to obtain a complete data set from 15:05 to 16:50 UT, when the sunspot was located at a heliocentric angle of $\theta=33^{\circ}(\mu=\cos \theta=0.84)$. The images cover an area of $58 \times 57^{\prime \prime}$ with a spatial sampling of 0. . $^{\prime} 059$ pixel $^{-1}$. More details of the observations can be found in Watanabe et al. (2012).

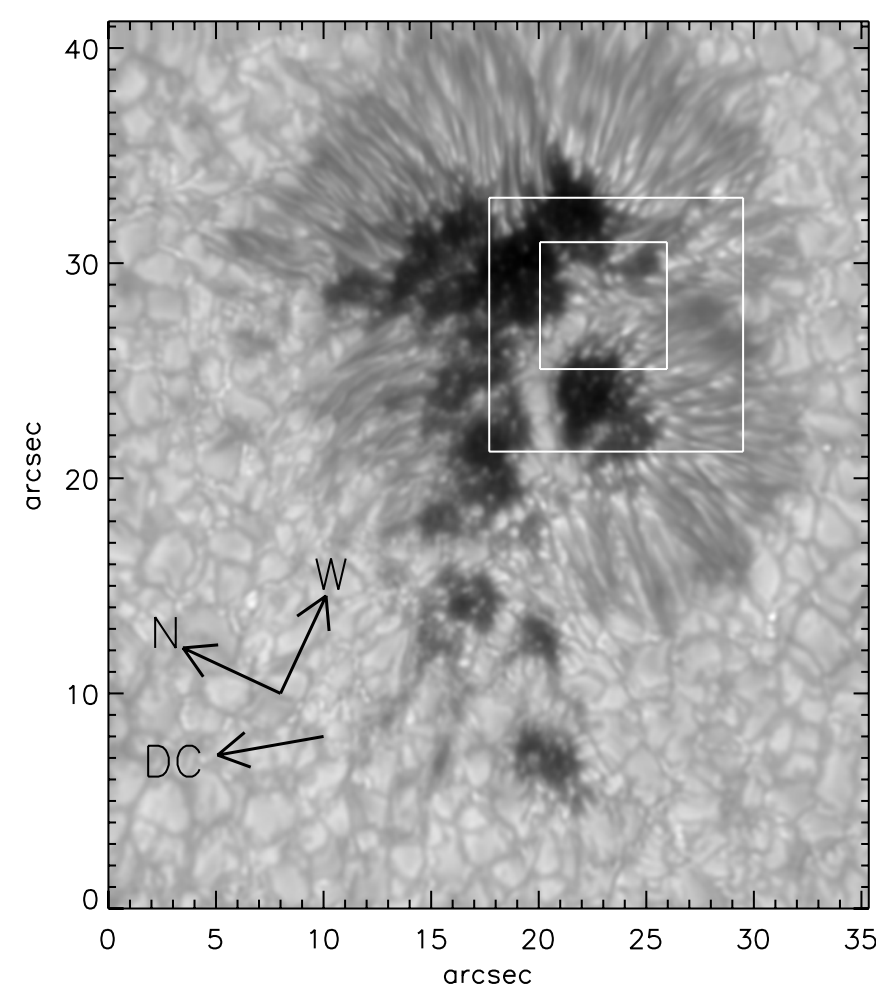

Fig. 1. Continuum image of the leading sunspot of AR 11024 at $630 \mathrm{~nm}$ on 2009 July 5, 16:44 UT. The large and small white boxes correspond to the field of view shown in Figs. 2 and 3, respectively. DC indicates the disc centre position.

Figure 1 shows a continuum image of the main spot of AR 11024 recorded through the CRISP narrowband channel. A granular LB is clearly seen, anchored to the eastern and southern penumbra. The spot shows disruptions in the penumbra near the eastern part of the LB. The region chosen for analysis consists of the LB and a portion of the limbside penumbra (boxed area in the figure).

\section{Results}

\subsection{Strong photospheric flows and chromospheric activity}

Figure 2 presents a photospheric Dopplergram of the LB and its surroundings at 16:44 UT (first panel, bottom row). Dopplergrams were computed from bisectors at different intensity levels of the FeI $630.15 \mathrm{~nm}$ line using the procedure described in Rouppe van der Voort et al. (2010), with the dark umbral regions serving as a reference for the absolute wavelength scale. The Dopplergram shows a patch of blueshifts of $\sim 1.5 \mathrm{~km} \mathrm{~s}^{-1}$ next to a strong redshift of $3 \mathrm{~km} \mathrm{~s}^{-1}$ on the entrance of the LB. Blueshifts in other parts of the LB are typically $700 \mathrm{~m} \mathrm{~s}^{-1}$, while the limbside penumbra displays redshifts ranging from 1 to $2 \mathrm{~km} \mathrm{~s}^{-1}$ associated with the Evershed flow (Evershed 1909). The patches of strong blueshifts and redshifts (hereafter referred to as the blue and red patches, respectively) have an area of $\sim 0.8 \operatorname{arcsec}^{2}$ and are separated by about $11^{\prime \prime} 6$.

To determine the magnetic field configuration associated with these photospheric flows, we inverted the Fe I $630 \mathrm{~nm}$ Stokes profiles observed in the region of interest with the SIR code (Stokes Inversion based on Response functions; Ruiz Cobo \& del Toro Iniesta 1992). A single magnetic component in each pixel was used, perturbing temperatures, magnetic field strengths and line-of-sight (LOS) velocities at two nodes. The 


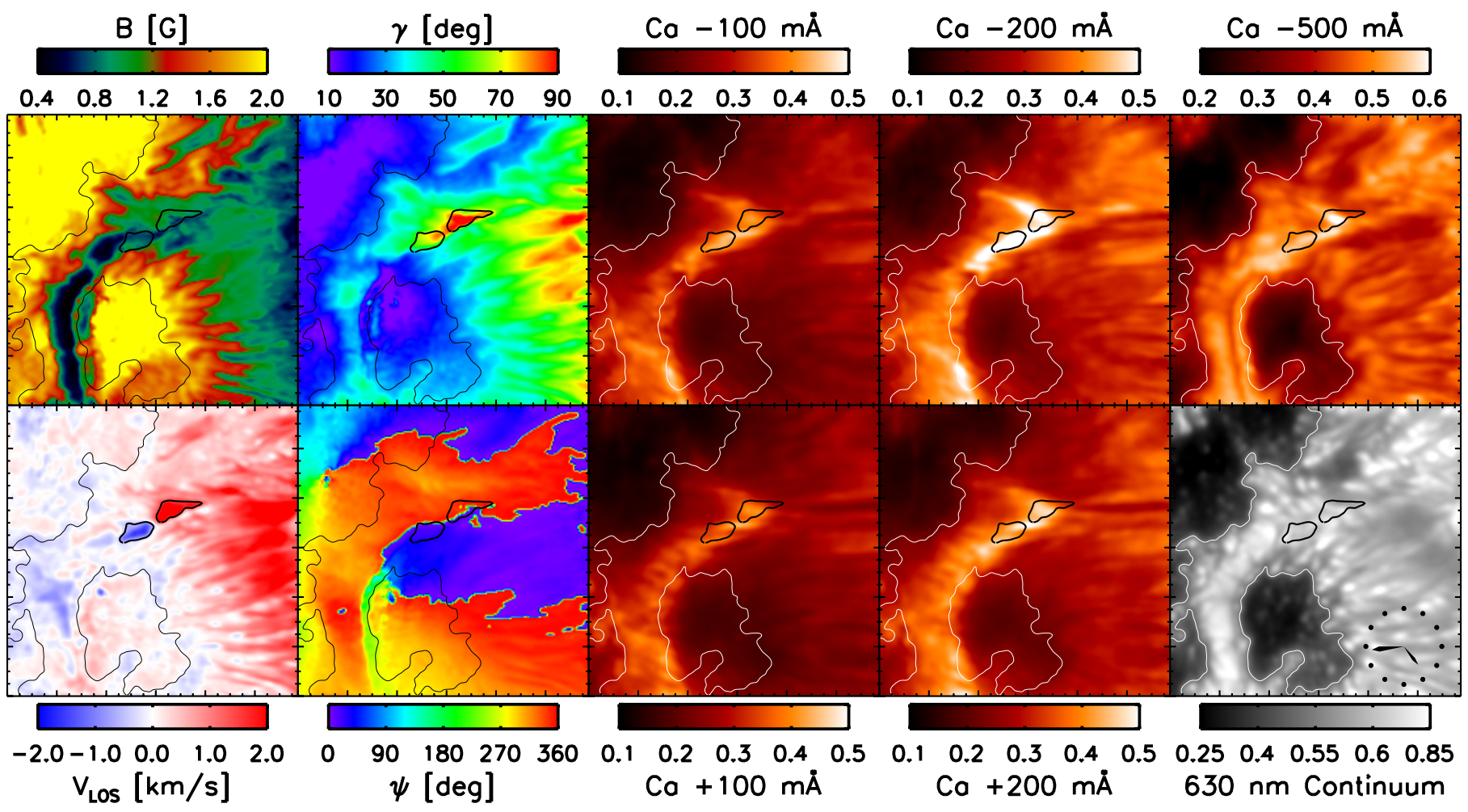

Fig. 2. Physical parameters and Ca II intensity filtergrams of the LB and its surroundings. The $12^{\prime \prime} \times 12^{\prime \prime}$ field of view corresponds to the large square indicated in Fig. 1. Column 1: magnetic field strength (top) and Dopplergram from bisectors at the 70\% intensity level (bottom). The field strength map corresponds to an optical depth of $\log \tau=-1$.0. Column 2: field inclination (top) and azimuth (bottom) in the local reference frame. Inclinations of $0^{\circ}$ and $180^{\circ}$ imply that the field is directed away from and into the solar surface, respectively. Zero azimuth is along the positive $x$-axis and increases in the counter-clockwise direction. Columns 3-5: intensity filtergrams at different wavelength positions of the Ca II $854.2 \mathrm{~nm}$ line and the continuum image at $630 \mathrm{~nm}$. All images have been scaled according to their respective colour bars. The maps correspond to scan number 97 acquired at 16:44 UT. The temporal evolution is shown in a movie available online.

field inclination and azimuth were kept constant with height. We also considered a constant value of macroturbulence $\left(0.5 \mathrm{~km} \mathrm{~s}^{-1}\right)$ and zero stray light in all pixels. The field inclination and azimuth were subsequently transformed to the local reference frame. They are shown along with the field strength in Fig. 2.

The blue and red patches have field strengths of about $820 \mathrm{G}$ and $900 \mathrm{G}$, respectively, whereas the typical field strength in the rest of the LB, particularly along its axis, is of the order of $400 \mathrm{G}$ and therefore much weaker. The field strength map corresponds to an optical depth of $\log \tau=-1.0$ since the response of the Fe I $630 \mathrm{~nm}$ lines to magnetic fields is maximum around that height (Cabrera Solana et al. 2005).

Figure 2 also shows that the red patch consists of nearly horizontal fields (inclinations of $90^{\circ}$ ) with the polarity changing sign in several pixels. These highly inclined fields extend partially into the blue patch where the mean inclination is $62^{\circ}$ but individual pixels reach a maximum of $83^{\circ}$. By comparison, the magnetic field in the arm of the LB is relatively vertical, with inclinations of $40^{\circ}$. The two patches sit close to a section of the sunspot where the field azimuth on either side confluences by some $40^{\circ}$. This arises from the two azimuth centres located in the two umbral cores partitioned by the LB.

Columns 3-5 in Fig. 2 show intensity filtergrams at different wavelength positions within the Ca II $854.2 \mathrm{~nm}$ line. The blue and red patches are co-spatial with enhanced brightness that is stronger in the blue wing compared with the red wing, particularly near the knee of the line. The brightenings are also observed at other positions of the line wing (e.g. $-500 \mathrm{~m} \AA$ ), but they are more localised. The conspicuous $\mathrm{V}$-shaped chromospheric brightening seen at $-200 \mathrm{~m} \AA$ (fourth column, top panel) has an intensity of $0.57 I_{\mathrm{QS}}$ and is nearly $20 \%$ brighter than the neighbourhood.

\subsection{Temporal evolution of the flow structures}

Figure 3 shows the development of the blueshifts and redshifts in the LB and the associated chromospheric brightenings during the last 30 min of observations. The sequence starts at minute 75 (16:20 UT) with the red patch already present two minutes earlier. The blue patch appears four minutes later and grows significantly from $0.07 \operatorname{arcsec}^{2}$ to $0.97 \operatorname{arcsec}^{2}$ in a time span of three minutes, becoming nearly as large as the red patch. Both patches remain next to each other, close to the entrance of the $\mathrm{LB}$, until the end of the sequence, implying lifetimes greater than $25 \mathrm{~min}$. The blue patch maintains an elliptical structure with its major axis nearly parallel to that of the LB. The movie accompanying Fig. 2 shows that the blue patch coincides with an elongated emerging granule (rows 2 and 3 of Fig. 3). The red patch lies near the end of this granule, which is interrupted by the penumbral filaments. At that position, the radial arrangement of the penumbra further out is also disrupted. The two patches do not exhibit significant proper motions, but evolve in situ with their respective centroids spaced apart by about 1 .' 6 .

The sequence of field strength maps shows a small but discernible increase of field strength with the onset of the blueshifts and redshifts in the LB. This can be seen between rows 2 to 4 of Fig. 3. Interestingly, the field inclination bears a strong association with the photospheric blue- and redshifts. Rows 1 


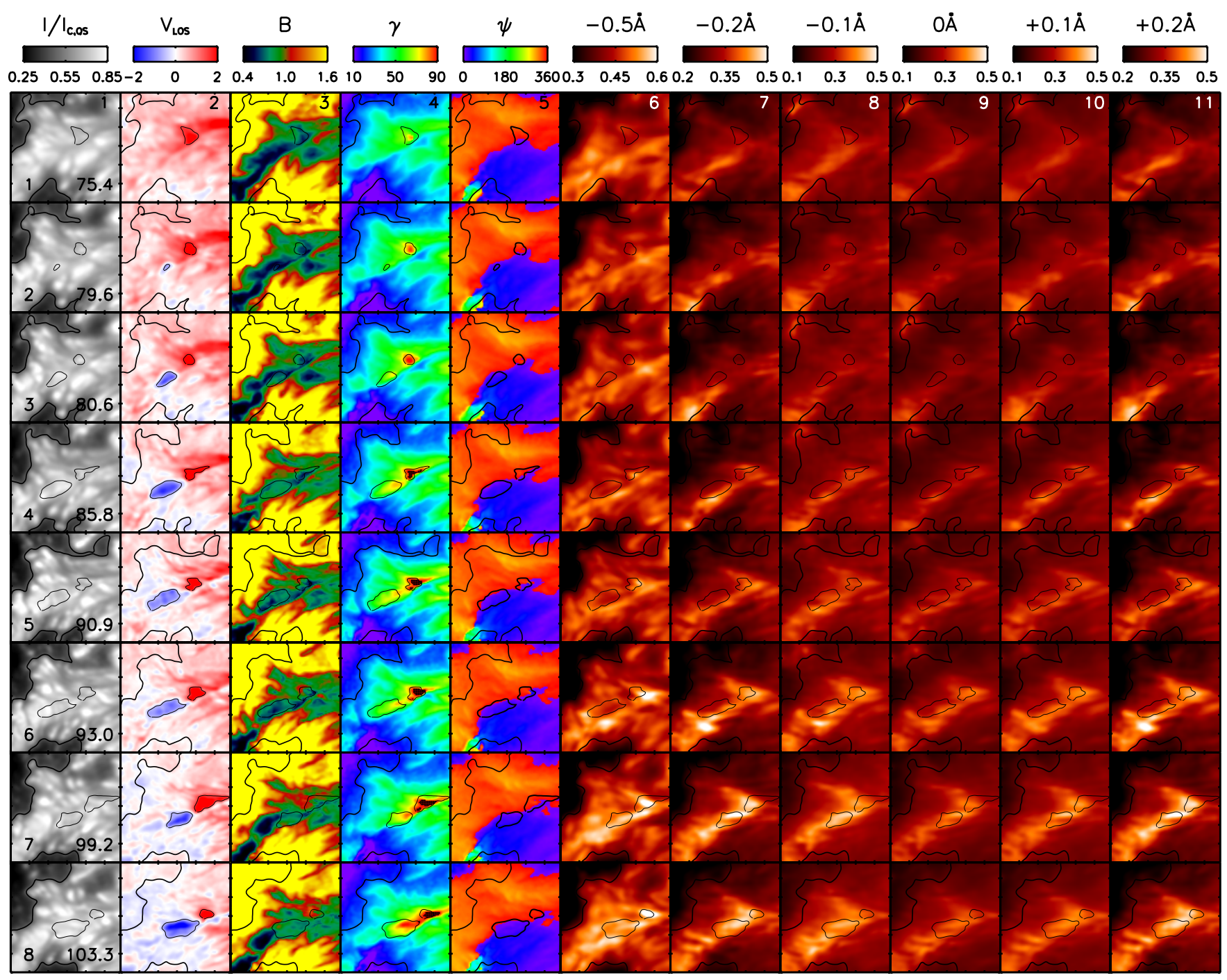

Fig. 3. Evolution of blueshifts and redshifts in the LB and their corresponding signature in the chromosphere. The $6^{\prime \prime} \times 6^{\prime \prime}$ field of view corresponds to the small square indicated in Fig. 1. Columns 1-5: continuum intensity at $630 \mathrm{~nm}$, LOS velocity, magnetic field strength, inclination, and azimuth, respectively. In Col. 4, all pixels exceeding an inclination of $90^{\circ}$ are coloured black. Columns 6-11: intensity filtergrams at different wavelength positions of the Ca II $854.2 \mathrm{~nm}$ line. All images have been scaled according to their respective colour bars. The first column gives the row numbers and times of the scan at the bottom of each panel. Column numbers are indicated at the top of each panel in row 1.

and 2 indicate that the red patch is an isolated concentration of magnetic fields that are more horizontal than the surroundings. The field inclination increases in the red patch until it changes polarity at minute 86 (black pixels in panel 4). The field also becomes more inclined in the blue patch as it evolves, but it never changes polarity. There are no significant variations in the field azimuth related to the evolution of either of the two patches.

Columns $6-11$ of Fig. 3 indicate the temporal evolution of the $\mathrm{Ca}$ intensity at different wavelength positions sampling the region between the mid-photosphere to the low chromosphere. The Ca filtergrams at $\pm 500 \mathrm{~m} \AA$ exhibit isolated bright patches in different parts of the FOV, which do not appear to be related to the development of the photospheric flows. The chromospheric response to the redshifts and blueshifts is clearly seen at minute 93 (row 6) in the form of the $\mathrm{V}$-shaped brightening described earlier. The brightening starts between minute 83 and 90 (rows 4 and 5). It is observed at all wavelength positions, but is relatively more intense in the blue wing. In the line core (Col. 9), this feature is comparatively subdued, although discernible. The strong chromospheric brightening persists until the end of the observations.

The left panels of Fig. 4 show the mean value of various parameters averaged within the blue patch as a function of time. The mean velocity reaches a peak of $1.6 \mathrm{~km} \mathrm{~s}^{-1}$ nearly three minutes after the emergence of the blueshifts in the LB. At this time, the maximum velocity in the patch is $2.6 \mathrm{~km} \mathrm{~s}^{-1}$. For comparison, the maximum velocity in the red patch is about $4.5 \mathrm{~km} \mathrm{~s}^{-1}$ at minute 94, while the mean velocity is $2 \mathrm{~km} \mathrm{~s}^{-1}$ (not shown). The increase in velocity in the blue patch is accompanied by enhancements of the field strength and inclination of $400 \mathrm{G}$ and $25^{\circ}$, respectively. The mean value of the magnetic field and inclination in the patch is $1.3 \mathrm{kG}$ and $70^{\circ}$ at the end of the sequence. The red patch is some $500 \mathrm{G}$ stronger, but the field strength variation is similar.

The right panels of Fig. 4 depict the mean $\mathrm{Ca}$ intensity at different wavelength positions. The mean $\mathrm{Ca}$ intensity peaks around minute 96 , nearly $17 \mathrm{~min}$ after the blue patch emerges in the photosphere. All the wavelength positions exhibit an 
R. E. Louis et al.: Small-scale magnetic flux emergence in a sunspot light bridge
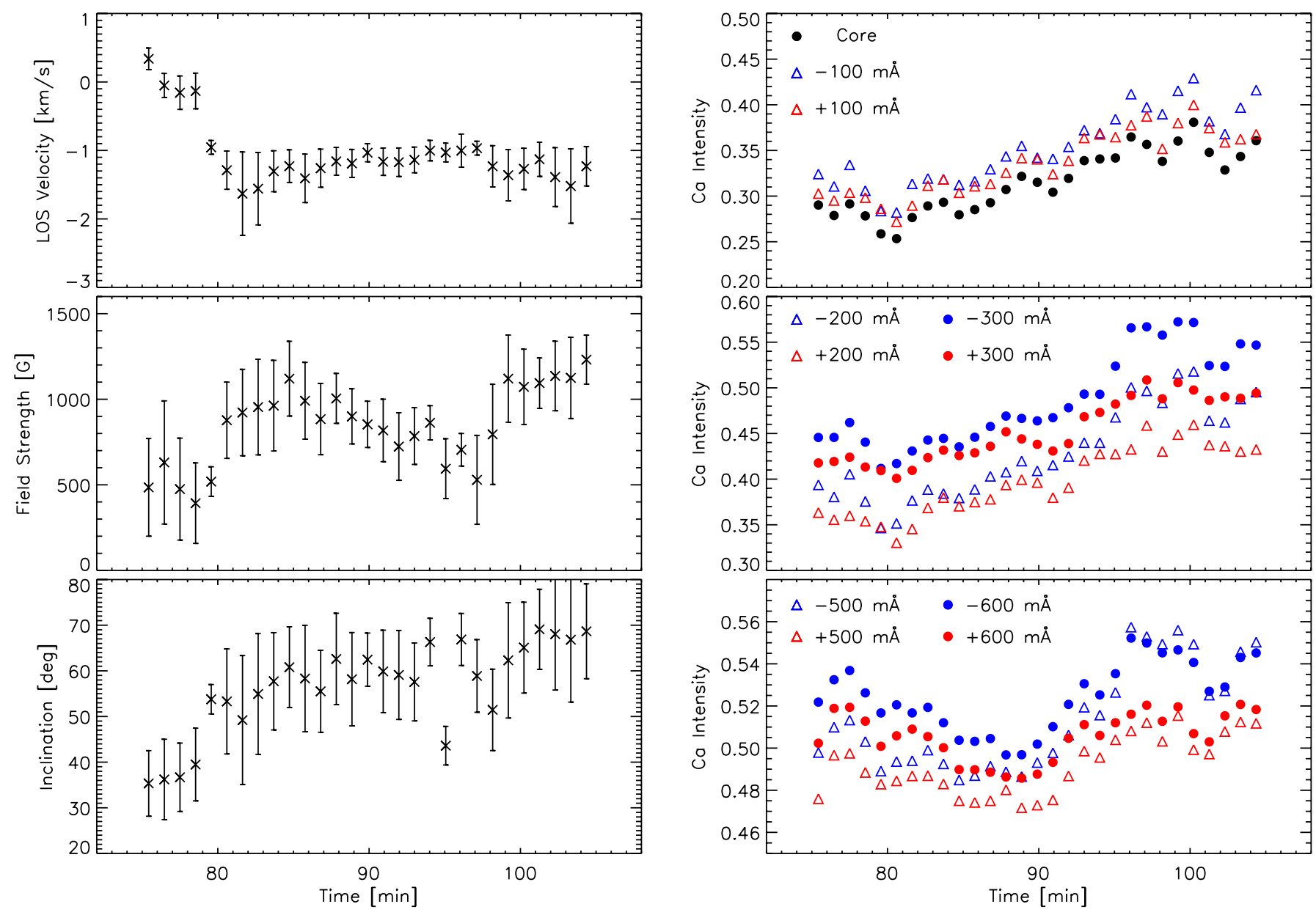

Fig. 4. Temporal variation of physical parameters averaged over the blue patch. The set of panels on the right correspond to the mean Ca intensity at different wavelength positions. The vertical bars in the left panels represent the standard deviation of the individual values entering the mean.

intensity increase, although it is stronger in the blue wing compared to the red wing. While the increase is gradual for the spectral range from -300 to $+300 \mathrm{~m} \AA$, further out in the line it is steeper (bottom right panel of Fig. 4). The intensity increases sharply from minute 94 to 96 , particularly at -300 and $-200 \mathrm{m \AA}$, by $8 \%$ and $6 \%$, respectively. In general, the mean blue wing intensity exceeds the red wing intensity by about $5-10 \%$, depending on the wavelength position. Around the line core, the intensity increases by some $15 \%$ (top right panel) while in the 200-300 $\mathrm{m} \AA$ range the increase is $20 \%$ (middle right panel). Finally, towards the photosphere (bottom right panel) the increase is relatively weaker $(6-10 \%)$.

\subsection{Spectral characteristics of Ca brightening}

In this section, we describe the spectral characteristics of the chromospheric brightening at the location of the photospheric blueshifts and redshifts. The top panel of Fig. 5 shows a Ca filtergram at $-200 \mathrm{~m} \AA$ from line centre at minute 99 . The white and black contours enclose the umbra and penumbra of the sunspot. The blue and red contours show the blue and red patches, respectively. The bottom panel shows the average Ca intensity profile emerging from them, as well as from the QS, at minute 99. For comparison, the average profile in a small region next to the brightening is also shown.
The Ca II $854.2 \mathrm{~nm}$ profile at the position of the blueshifts shows a strong emission feature in the blue wing of the line, around -400 and $-300 \mathrm{~m} \AA$. A similar feature can be observed in the red wing, although it is less prominent. Only a few pixels exhibit comparable emission peaks in the red patch. The average profile shows hints of emission in the blue wing and higher intensities than in the red wing, but no strong peaks are observed. Another important fact is that the line core is not Doppler shifted in either of the two patches. These Ca II $854.2 \mathrm{~nm}$ profiles are rather different from those recorded outside the intense brightening (triangles), which resemble the QS profile except for an overall offset. Profiles with asymmetric emission in the line wing have been seen in observations (e.g., Ortiz et al. 2014) and in numerical simulations (Leenaarts et al. 2009).

Figure 6 shows the temporal evolution of the Stokes $I$ and $V$ profiles of the Ca II $854.2 \mathrm{~nm}$ line in the two patches. We have selected the pixel with the highest average intensity between -300 and $-400 \mathrm{~m} \AA$. Prior to the appearance of the blueshifts in the photosphere, the $\mathrm{Ca}$ intensity profiles look normal. Around minute 84 , the profile from the blue patch starts to develop a knee in the blue wing while the profile from the red patch remains unaffected. The emission feature in the wings is seen around minute 89 and 95 in the two patches, respectively. Both patches show asymmetric emission that persists until the end of the sequence. There are instances in which the emission in the red patch is stronger than that in the blue patch (e.g. at 

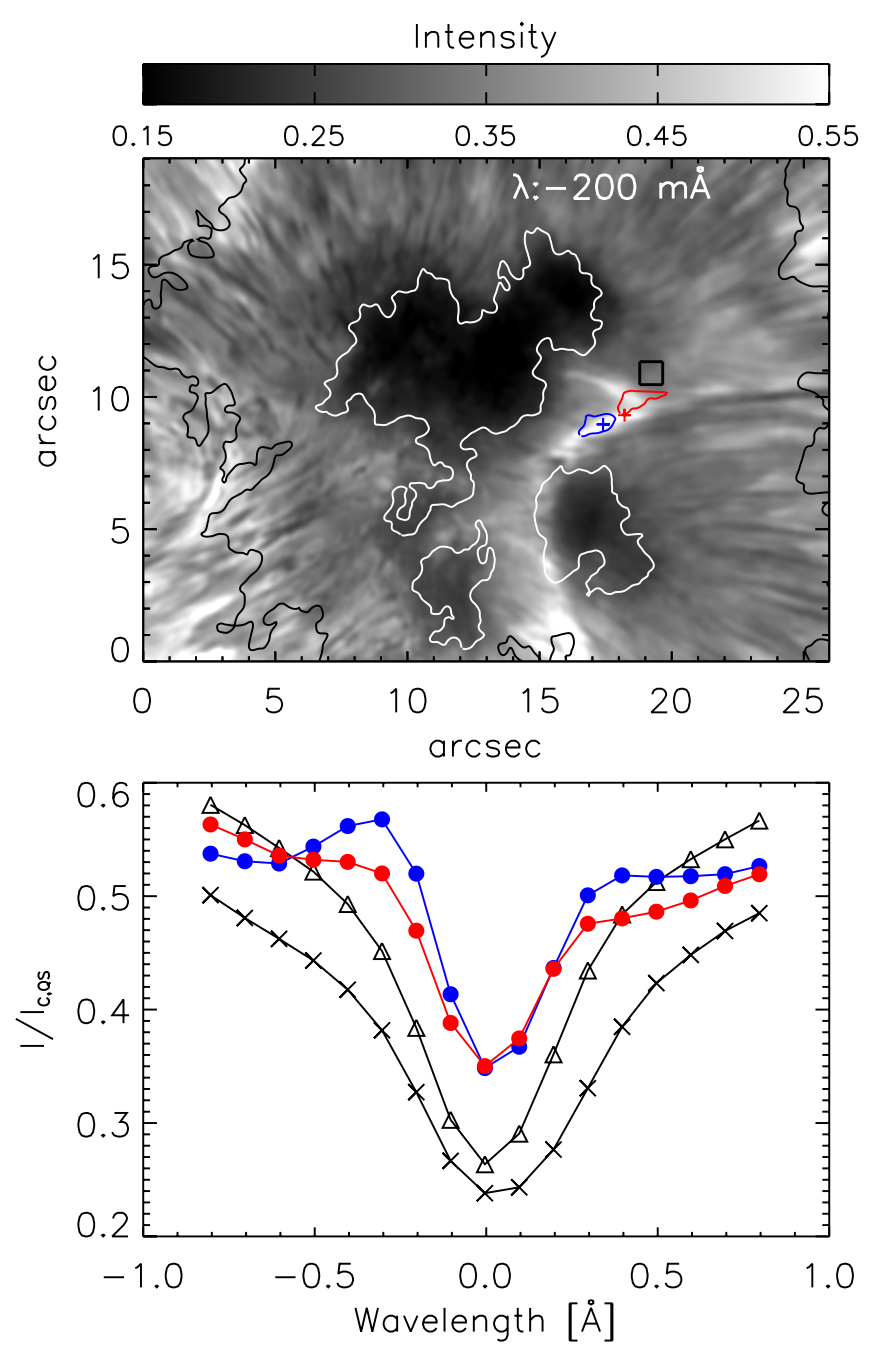

Fig. 5. Intensity profile at the location of the brightening. Top panel: Ca filtergram at $-200 \mathrm{~m} \AA$ from line centre at minute 99 . The blue and red contours indicate the photospheric blueshifts and redshifts. The blue and red plus symbols refer to the pixels whose intensity profiles are shown in Fig. 6. Bottom panel: mean Ca intensity profile in the quiet Sun (crosses), blue patch (blue circles), red patch (red circles) and thick black square close to the brightening (triangles). The average profiles correspond to the scan at minute 99 .

minute 95 and 97). As already mentioned, the core of the line is not shifted significantly in any of the patches.

The Stokes $V$ profiles show variations in asymmetry and amplitude that seem to be unrelated to the development of flows in the photosphere. In any case, the sign of the profiles remains unchanged during the observations in both patches.

\subsection{Temperature and velocity in the chromosphere}

\subsubsection{Stokes inversions}

We now examine the physical conditions in the chromosphere associated with the brightenings that occur above the photospheric blueshifts and redshifts. The conditions prior to the appearance of the brightenings are also investigated to determine how the chromosphere is modified from quiescence.

In Fig. 7 we show the Stokes profiles observed in the photosphere and the chromosphere before (Cols. 1 and 2) and during the brightening (Cols. 3 and 4 ). The profiles come from the pixel in the blue patch where the intensity between -300 and $-400 \mathrm{~m} \AA$ is highest (indicated with a cross in Fig. 5) at 16:19 UT (minute 74) and 16:44 UT (minute 99). They are representative of the typical profiles encountered in this patch. As mentioned before, the $\mathrm{Ca}$ intensity profile is normal during the quiescent phase and subsequently exhibits an asymmetric emission in the line wing which is the source of the observed brightenings. The Ca II $854.2 \mathrm{~nm}$ Stokes $V$ profiles do not change sign between the two instances of time. The chromospheric $Q$ and $U$ signals are quite noisy, irrespective of the presence or absence of the brightenings. The Fe I lines show significant changes, particularly in Stokes $I$ and $V$. The $I$ profiles are at rest during the quiescent phase and clearly blueshifted 25 min later. Furthermore, the Stokes $V$ profiles, which initially have normal, anti-symmetric lobes, change to highly asymmetric lobes with reversed signs. The sign reversal is a projection effect due to the appearance of highly inclined fields (see below).

To infer the temperature and velocity stratification in the chromosphere, we employed the Non-LTE Inversion COde based on the Lorien Engine (NICOLE; Socas-Navarro et al. 2015). The four Stokes profiles of the Ca II $854.2 \mathrm{~nm}$ line were inverted along with the two Fe lines (so as to cover a large height range and to provide suitable boundary conditions for the NLTE calculations of Ca II $854.2 \mathrm{~nm}$ ). We ran NICOLE with two cycles, allowing a maximum of 25 iterations per cycle. The FALC model (Fontenla et al. 1993) served as an initial guess atmosphere, and the parameters resulting from the first cycle were used to start the second cycle. In the first cycle, temperature $(T)$, LOS velocity $\left(V_{\mathrm{LOS}}\right)$, LOS magnetic field $\left(B_{\mathrm{LOS}}\right)$, and the two transverse components of the magnetic field $\left(B_{\mathrm{x}}, B_{\mathrm{y}}\right)$ were perturbed with $2,1,2$, and 1 nodes, respectively. In the second cycle, the number of nodes was changed to $6,3,3$, and 2 , plus 3 nodes for microturbulence. To invert the profiles that show the Ca brightening, the number of nodes in $T$ and $V_{\text {LOS }}$ was increased to 8 and 4 , respectively. Stray light was included in the inversion, assumed to be wavelength-independent.

The solid lines in Fig. 7 show the best-fit profiles resulting from the inversions. They match the observed profiles quite well. With the exception of the continuum point, the fit to the Ca II $854.2 \mathrm{~nm}$ intensity profile seen during the quiescent phase is good. Also the asymmetric emission in the line wing is well reproduced, as is the nearly stationary line core during the brightening (top panel in Col. 3). The observed and best-fit Stokes $V$ profiles of the $\mathrm{Ca}$ line are in good agreement. In the photosphere, the inversions reproduce the sign and, to a great extent, the asymmetry of the Stokes $V$ signals associated with the blueshifts.

The model atmospheres returned by NICOLE are shown in Fig. 8. During the quiescent phase, the temperatures are slightly hotter than those in the FALC model, except near the continuum forming layer. There are redshifts of about $1.5 \mathrm{~km} \mathrm{~s}^{-1}$ at $\log \tau=-3.0$ and weaker motions in higher and lower layers. The magnetic field strength and inclination are nearly constant with height, reaching values of about $750 \mathrm{G}$ and $40^{\circ}$, respectively. At the time of the brightenings, the temperature stratification exhibits a clear enhancement between $\log \tau=-5.0$ and -3.0 , with an excess of some $720 \mathrm{~K}$ relative to the quiescent phase. The LOS velocity changes sign and shows maximum blueshifts of $3 \mathrm{~km} \mathrm{~s}^{-1}$ at $\log \tau=-2.3$ that decrease to zero towards $\log \tau=-6.0$. The chromospheric field does not undergo significant changes from the quiescent phase to the brightenings, contrary to the photospheric field: in the photosphere, the field strength increases by about $100 \mathrm{G}$ and becomes nearly horizontal (with inclinations of approximately $88^{\circ}$ to the local vertical). Above $\log \tau=-4.0$, i.e. beyond the temperature minimum 
R. E. Louis et al.: Small-scale magnetic flux emergence in a sunspot light bridge
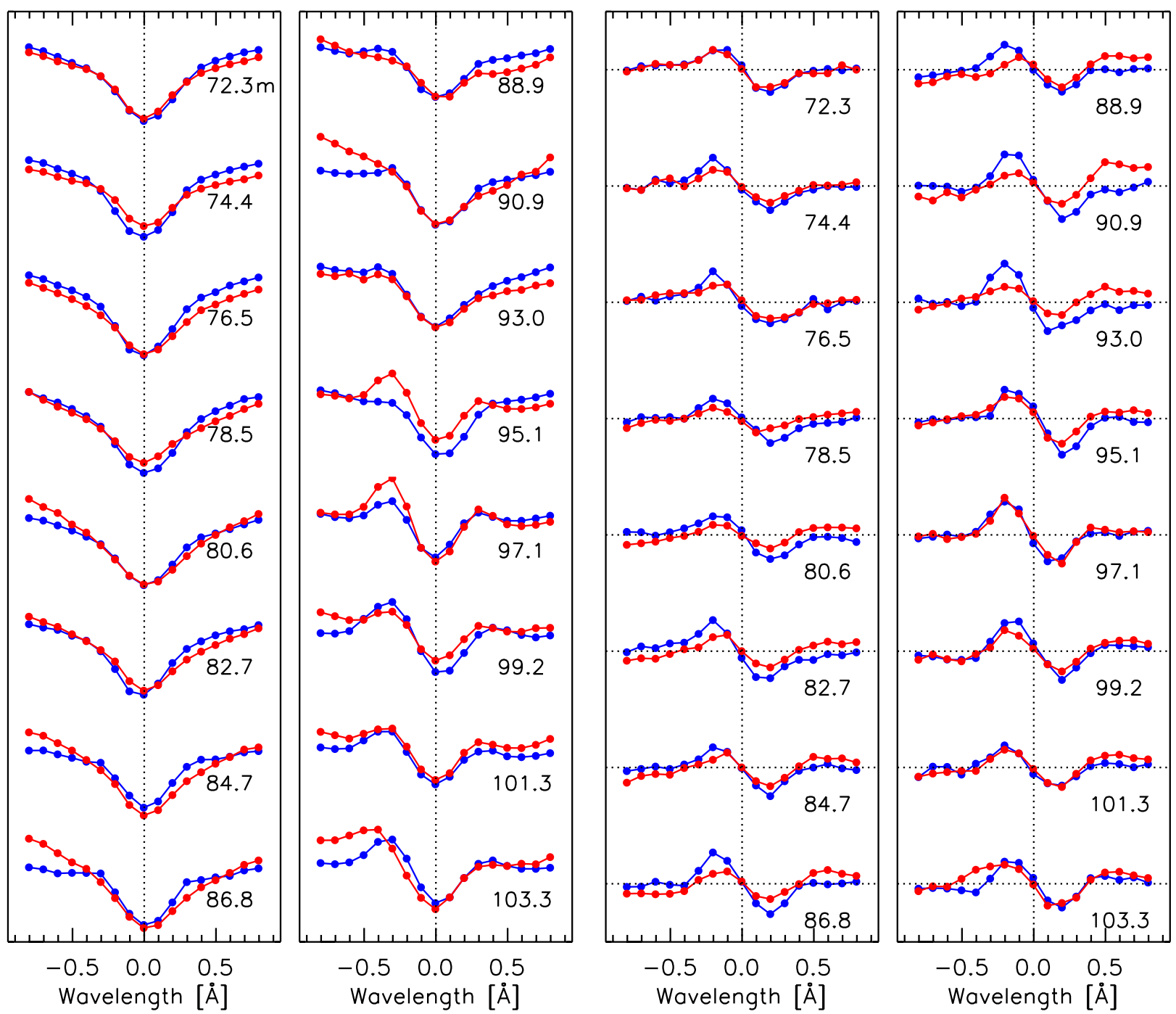

Fig. 6. Temporal evolution of Ca II $854.2 \mathrm{~nm}$ spectra in the blue and red patches (blue and red circles, respectively). Columns 1 and 2 correspond to Stokes $I$, while Cols. 3 and 4 correspond to Stokes $V$. Time increases from top to bottom and from left to right. The scaling of the $y$-axis in normalised intensity units is between $(0.2,0.7)$ and $(-0.05,0.05)$ for the two Stokes parameters. The profiles come from the pixel with the highest average intensity between -300 and $-400 \mathrm{~m} \AA$.

region, the field strength and inclination continue to be $750 \mathrm{G}$ and $40^{\circ}$ as in the quiescent phase.

To summarise, the blue patch in the LB is associated with rather vertical fields at the beginning. Later the fields become highly inclined in the photosphere, but do not change in the chromosphere. The brightening over the patch is due to asymmetric emission in the Ca II $854.2 \mathrm{~nm}$ line, caused by strong temperature enhancements near the upper photospheric/lower chromospheric layers, together with flows towards the observer of about $3 \mathrm{~km} \mathrm{~s}^{-1}$ in the mid-photosphere.

\subsubsection{Origin of the asymmetric Ca line-wing emission}

The model atmosphere derived from the inversion of the observed profiles reproduces the asymmetric emission that causes the brightenings above the photospheric blueshifts. In this section we further investigate the origin of such an asymmetry.

Temperatures and LOS velocities in high layers must play a more important role in producing the asymmetric emission than the magnetic field since the latter does not change dramatically with respect to the quiescent phase where the emission is absent.
To assess the influence of mass motions, we performed a spectral synthesis of the $\mathrm{Ca}$ and $\mathrm{Fe}$ lines setting to zero all the velocities in the model atmosphere returned by NICOLE. The result is shown in Fig. 9. Without velocities, the asymmetric emission in the $\mathrm{Ca}$ line disappears and we see symmetric emission peaks in both line wings. The Stokes $V$ profiles remain more or less unaffected, however, the lack of plasma motions shifts the Fe lines away from their position. Moreover, the red and blue Stokes $V$ lobes become nearly antisymmetric, strongly deviating from the observed profiles. Therefore, a gradient in the LOS velocity is needed to account for the shape of the circular polarization profiles recorded in the photosphere (Illing et al. 1975).

This simple exercise demonstrates that (1) an enhancement in temperature alone produces emission that is symmetric in both wings of the $\mathrm{Ca}$ line; and (ii) the LOS velocity serves to enhance the emission in one wing of the line, attenuating it in the other. Thus, to generate the spectral signatures observed at the location of the chromospheric brightenings, a combination of temperature and LOS velocity perturbations is necessary.

We now investigate the type of temperature enhancements required to produce symmetric line wing emission in the first 

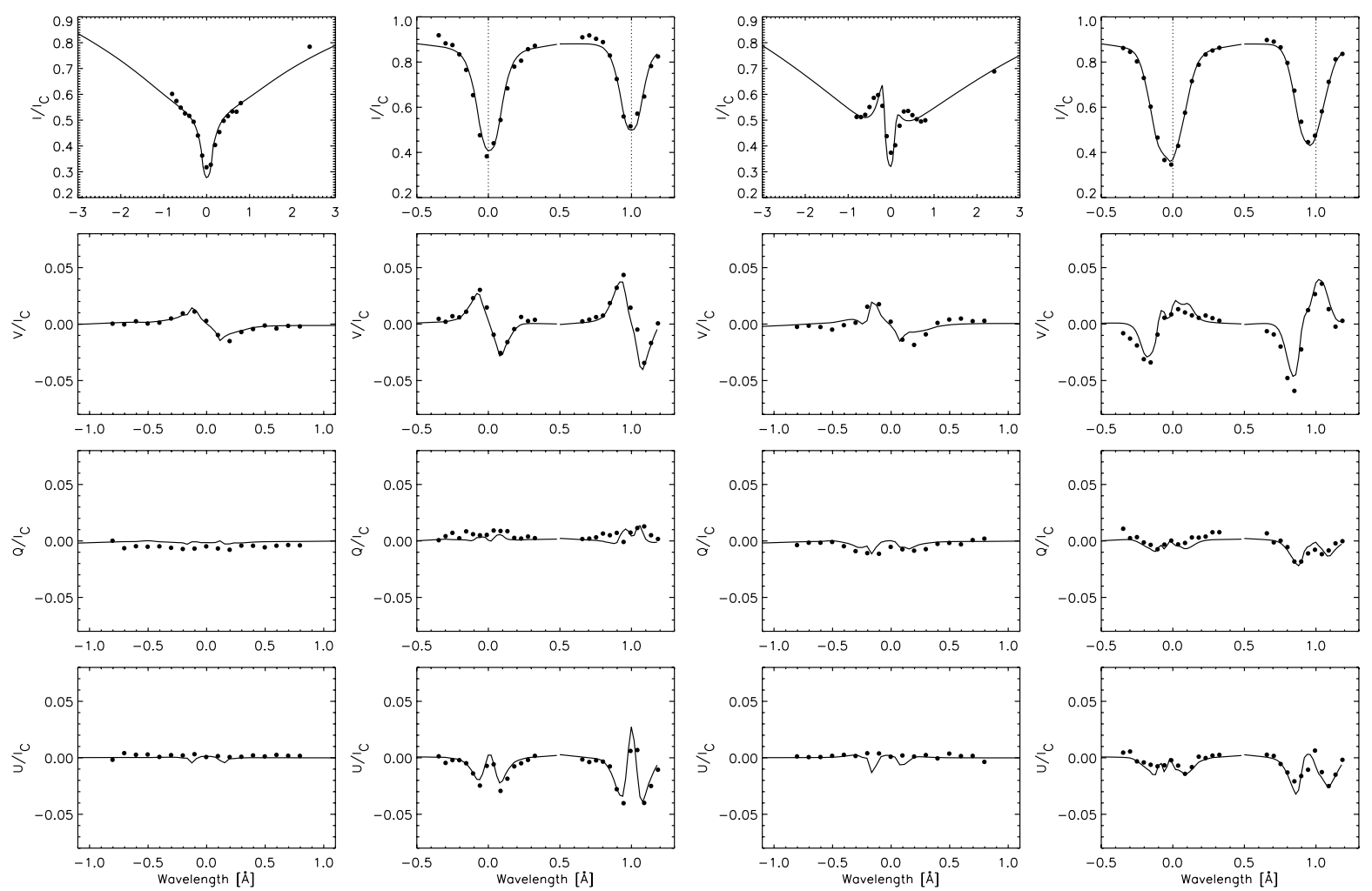

Fig. 7. Results from NICOLE inversions. Columns 1 and 2: Stokes profiles of the Ca and Fe lines, respectively, for the case when there was no brightening in the LB. Columns 3 and 4: same as Cols. 1 and 2, except for the case when the brightening was observed. Filled circles and solid lines represent observed and synthetic spectra, respectively.

place. To this end, we synthesised the Ca line, modelling the temperature enhancement as a Gaussian perturbation and changing its width, position, and amplitude. We used the FALC model as the reference atmosphere in these tests. The width of the temperature perturbation, $\sigma$, was made to vary from $0.4-0.6$ in units of $\log \tau$, the position was set between $-5.0 \leq \log \tau \leq-3.0$ and its amplitude was varied in the range $0-2000 \mathrm{~K}$. The temperature minimum is around $\log \tau=-3.8$ in the FALC model (Cauzzi et al. 2008). For simplicity, all the velocities were set to zero.

Figure 10 shows synthetic Ca II $854.2 \mathrm{~nm}$ line profiles for temperature perturbations of $+1000 \mathrm{~K}$ and various widths located at different optical depths. From these calculations we find that the strength of the emission depends on the position of the perturbation: the deeper the perturbation below the temperature minimum, the stronger the emission. This result is somewhat expected because the coupling of the line source function to the local temperature is usually weak (or non-existent) in the chromosphere and strong in the photosphere (see de la Cruz Rodríguez et al. 2013). Another obvious effect of perturbations in deeper layers is that the intensity ratio between the core and the wings becomes more pronounced, because the intensity of the wings is greatly enhanced.

The width of the perturbation seems to affect the wing intensity close to the knee-like feature of the line. Wider perturbations increase the overall wing intensity, but the effect is significantly smaller than that induced by the height of the perturbation.

As a final test, we perturbed the reference model using a linear temperature increase with height. The results show that there is no symmetric emissions in the line wing but the line exhibits a knee. Moreover, such a perturbation enhances the overall spectral line intensity. Increasing the temperature gradient also increases the wing to core intensity ratio.
From these tests we conclude that the temperature perturbation needed to produce emission in the wings of the Ca II line at the level shown in Fig. 9 (top left panel, solid line) cannot be located much higher than $\log \tau \sim-4$ or much deeper than $\log \tau \sim-2$. That is, the brightening appears to be an upper photospheric rather than a chromospheric phenomenon. Additionally, the temperature enhancement must extend over a minimum range of optical depths to lead to measurable effects on the emergent profiles. For a Gaussian perturbation of $1000 \mathrm{~K}$ located at $\log \tau=-3.5$, the minimum width is $\Delta \log \tau=0.6$.

\section{Discussion}

The photospheric LOS velocity maps obtained from the inversion of the observed Stokes profiles reveal the formation of nearby patches of upflowing and downflowing gas with opposite magnetic polarities in a sunspot LB. Shortly after these patches are formed, we observe chromospheric emission in the Ca II $854.2 \mathrm{~nm}$ line, which suggests that these events are physically connected. Here we try to identify the photospheric processes responsible for the flows and then interpret their subsequent coupling to the overlying chromosphere.

The first possibility that comes to mind is that the blueshifts and redshifts represent the source and termination points of an Evershed flow channel. The Evershed flow starts in bright penumbral grains, which are blueshifted in the limb-side penumbra and have speeds of about $0.5 \mathrm{~km} \mathrm{~s}^{-1}$ (Rimmele \& Marino 2006). The blueshifts we have observed are clearly stronger and, in addition, they coincide with granular and not filamentary structures. Also, we do not see clear proper motions of any of the patches, whereas it is known that the termination points of the Evershed flow move radially outwards. 
R. E. Louis et al.: Small-scale magnetic flux emergence in a sunspot light bridge
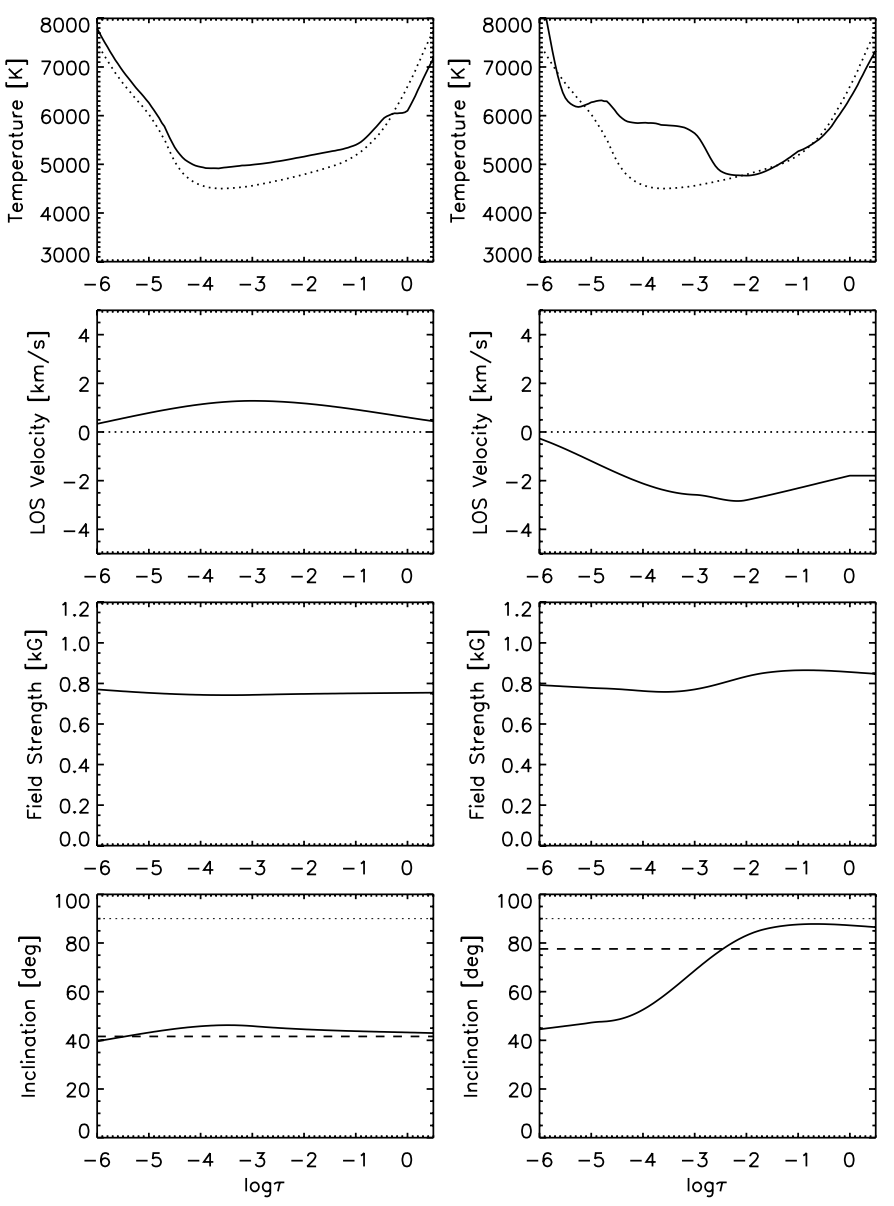

Fig. 8. Atmospheric parameters inferred from the NICOLE inversions (solid lines). Left: quiescent phase. Right: brightening phase. The dashed lines show the values obtained from the SIR inversions. Dotted lines represent the FALC temperatures, zero velocities, and horizontal fields in the respective panels.

A scenario compatible with the observations is that of a small-scale $\Omega$-loop that emerges in the LB and develops a siphon flow while rising through the atmosphere. The blue- and redshifted patches conform to a simple bipolar structure, which is magnetically connected by a vector field pointing from the blueshifts to the redshifts. The polarity of the magnetic field is positive (upwards) in the blue patch and negative (downwards) in the red patch. A siphon flow should develop along the loop because the red patch shows stronger fields $(\sim 400 \mathrm{G})$ and therefore the gas pressure is significantly decreased there. This pressure imbalance between the footpoints causes the gas to flow from the blue to the red patch. The loop footpoints do not separate during the sequence, probably because the highly magnetised environment of the LB impedes the horizontal expansion of the loop. The photospheric blueshifts and redshifts are accompanied by a noticeable increase in field strength. Moreover, the blue patch coincides with an emerging, elongated granule in the LB. Taken together, this suggests that we have witnessed a small-scale flux emergence event in the LB.

According to our results, the magnetic field in the footpoints is rather inclined and therefore the $\Omega$-loop must have a flat magnetic field topology. Figure 11 shows a sketch of the magnetic field orientation along the loop for some of the time steps. As the loop evolves, the field remains dominantly horizontal, only changing polarity in the redshifted footpoint. This is significantly different from quiet-Sun flux emergence, where
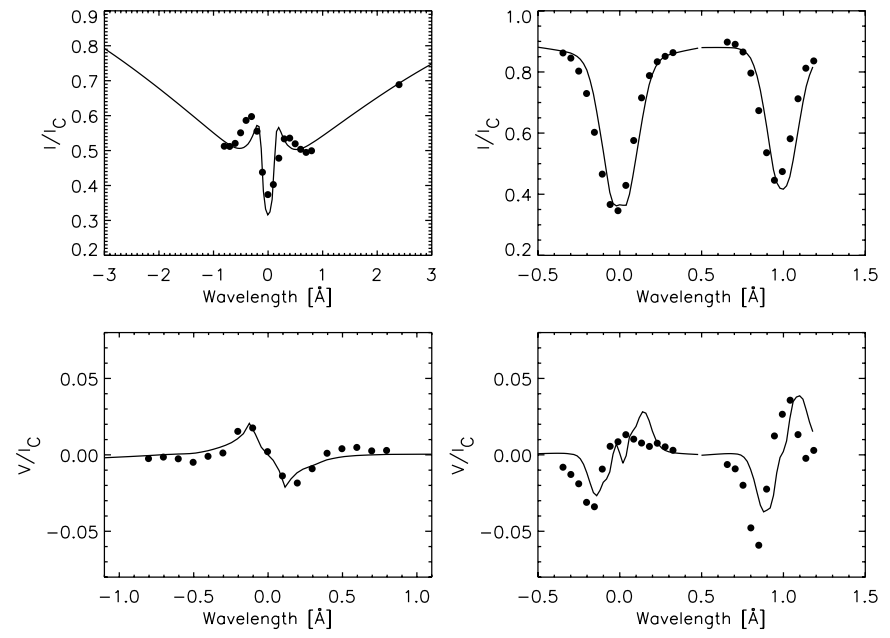

Fig. 9. Synthetic profiles (solid) emerging from the model atmosphere returned by NICOLE with zero velocities. The other parameters remain unchanged. The observed profiles are shown with filled circles.

the field becomes more vertical as the loop rises in the atmosphere (Martínez González \& Bellot Rubio 2009). Light bridges are known to be structures where the umbra is subject to convective instabilities (Jurčák et al. 2006; Louis et al. 2012). The granular morphology of the LB thus offers a natural location for the emergence of small-scale magnetic fields, aided by the upflows of convective cells. The emerging fields are highly inclined or even horizontal, suggesting that the strong sunspot fields around and above the loop influence the way in which they emerge into the LB. This could explain the asynchronous appearance of the footpoints of the loop.

The magnetic fields associated with the loop continue their ascent into the chromosphere where strong brightenings are observed 17 min after the development of the blueshifts. Compared with earlier LB studies based on Ca II H broadband imaging, we have the entire $\mathrm{Ca}$ II 8542 profile. The emission is located in the blue wing of the line, without an obvious Doppler shift of the line core. Light bridges are known to exhibit dynamic chromospheric phenomena as described in Sect. 1, with magnetic reconnection often cited as their driving mechanism (Berger \& Berdyugina 2003; Jurčák et al. 2006; Louis et al. 2009; Shimizu et al. 2009). In this case, magnetic reconnection is a plausible explanation for the observed brightening because the sunspot magnetic field over the LB runs across the redshifted patch, which should be favourable for component reconnection between the emerging field and the sunspot field.

We find that large enhancements in temperature and a moderate LOS velocity towards the blue, particularly in the region between the upper photosphere and the lower chromosphere (i.e., the minimum temperature region), are sufficient to produce the spectral features seen in Ca II $854.2 \mathrm{~nm}$ above the photospheric blueshifts. This layer of the solar atmosphere is extremely sensitive to fluctuations in the thermodynamic and kinematic parameters, all of which are directly or indirectly influenced by the magnetic field. The magnetic loop first emerges in the weakfield environment of the LB, but then it encounters the strong magnetic fields of the sunspot during its ascent, oriented at an angle with respect to the loop. These gradients in the magnetic field could lead to temperature enhancements through ohmic dissipation of electric currents (Solanki et al. 2003; Bourdin et al. 2013). We observe a gradual increase in the blue wing intensity close to the knee of the spectral line, followed by a steep rise. 

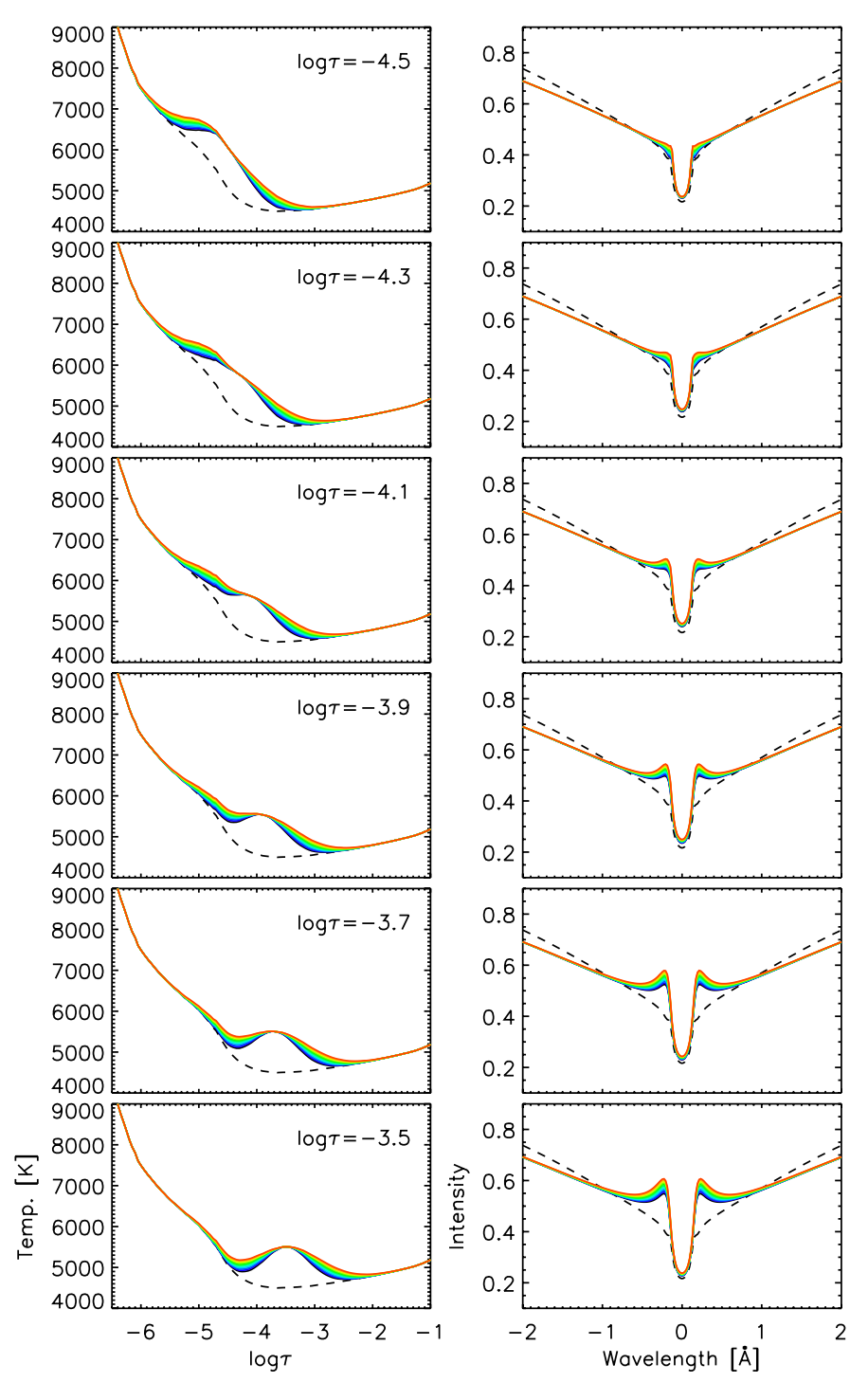

Fig. 10. Ca II $854.2 \mathrm{~nm}$ profiles emerging from the FALC model with perturbed temperatures. The left panels show a Gaussian temperature enhancement of $1000 \mathrm{~K}$ at various optical depths. In each panel, the colours represent different widths of the perturbation with blue and orange corresponding to $\Delta \log \tau=0.4$ and 0.6 , respectively. The dashed line represents the unperturbed FALC atmosphere. The panels on the right are the corresponding intensity profiles calculated with NICOLE. The black dashed line shows the unperturbed profiles.

The steep rise would be compatible with the idea of magnetic reconnection where the free energy is impulsively released.

The Ca II line core is unshifted, suggesting that the rising loop does not reach the chromosphere. In fact, the chromospheric magnetic field remains largely unaffected, unlike the photospheric field which becomes nearly horizontal during the emergence of the loop. Therefore, the emerging magnetic flux is confined to or trapped in the upper photospheric layers, possibly stopped by the overlying magnetic field of the sunspot. However, we do not rule out the possibility that the emerging magnetic field eventually reaches chromospheric heights, since the observing sequence ended before the flow structures disappeared.

The profiles analysed in the present study are not very different from those observed in flux emergence regions outside of sunspots (Ortiz et al. 2014). de la Cruz Rodríguez et al. (2015a) explained a similar (asymmetric) emission but in the red wing of
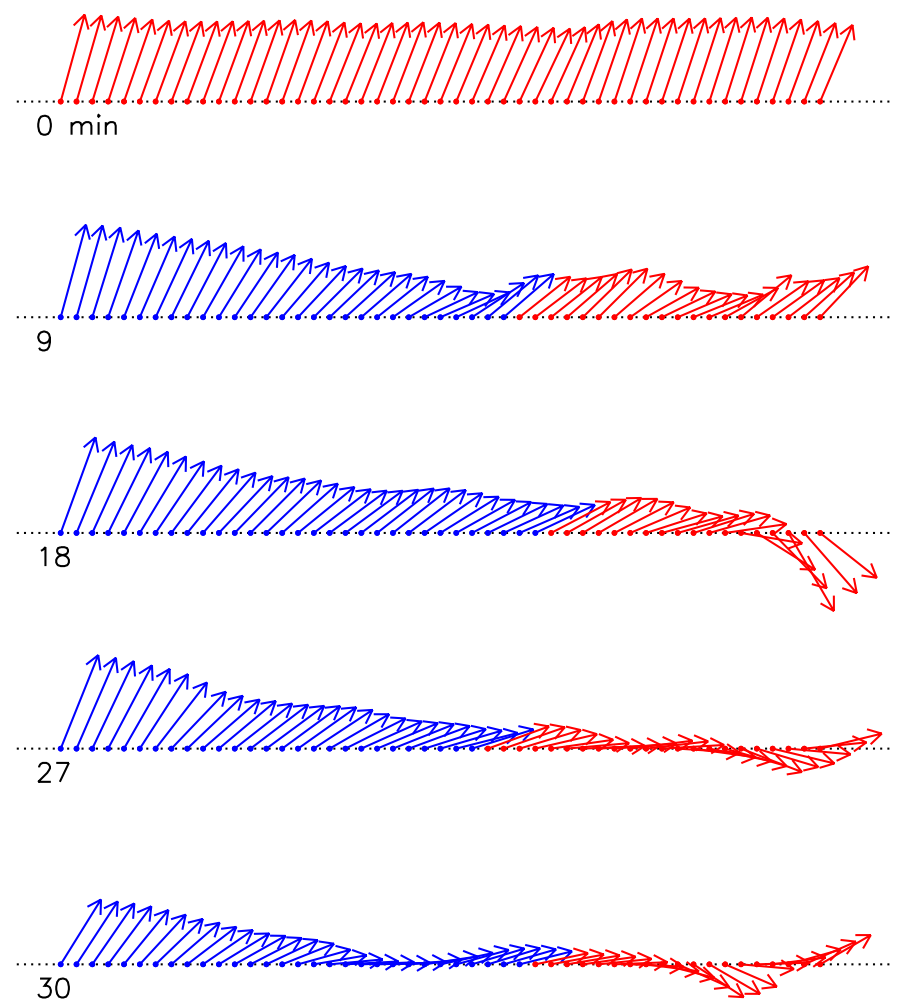

Fig. 11. Sketch of the flat $\Omega$-loop emerging in the light bridge. The arrows have been drawn for a cut passing along the blue and redshifted patches. The length and orientation of the arrows are proportional to the field strength and inclination inferred from the inversion. The start time corresponds to minute 72 of the time sequence.

the Ca II $854.2 \mathrm{~nm}$ line, within a flux-emerging region. In their observations and MHD simulations, the presence of a velocity field gradient shifts the absorption profile in the upper photosphere to shorter wavelengths. This allows photons to escape more easily in the red line wing, thus producing enhanced emission in that wing and the opposite effect in the other. The main difference here is that, although we also have upflowing material, the emission occurs in the blue line wing. Therefore, in our data the emission is obviously not caused by the same opacity effect reported by de la Cruz Rodríguez et al. (2015a), although the velocity field seems to be responsible for the observed asymmetry. There are also similarities with the Class-1 chromospheric transient events reported by Reardon et al. (2013) and Drews (2014). These events also exhibit a stationary line core and impulsive intensity increases that occur within a span of 3-4 min and are compatible with the sharp rise detected around minute 94.

In summary, we have interpreted the development of photospheric blue- and redshifts in a sunspot LB as the emergence of a small-scale, flat $\Omega$-loop, whose topology and evolution is influenced by the strongly magnetised environment of the sunspot. The Doppler shifts occurring in the two footpoints can be explained naturally in terms of a siphon flow, which develops as a consequence of a significant field strength imbalance. The ascending loop is probably trapped by the overlying sunspot field and the subsequent interaction is responsible for boosting the temperature near the upper photospheric/lower chromospheric layers, producing peculiar asymmetric emission in the blue wing of the CaII $854.2 \mathrm{~nm}$ line. Since we do not know the ultimate fate of this emission, the physical mechanism behind it remains an open question. By combining multiple spectral lines, which cover a large height range from the photosphere to the 
chromosphere, it is possible to determine the coupling between the different layers of the atmosphere. This will be the topic of further investigation.

Acknowledgements. Part of this work was done while R.E.L. was a Visiting Scientist at the Instituto de Astrofísica de Andalucía (CSIC), where he continued the analysis initiated at the Udaipur Solar Observatory as a Post Doctoral Fellow. Financial support by the German Science Foundation under grant DE 787/3-1, the European Commission's FP7 Capacities Programme under Grant Agreement number 312495 (SOLARNET project), and by the Spanish Ministerio de Economía y Competitividad through projects AYA2012-39636C06-05 and ESP2013-47349-C6-1-R (including a percentage from European FEDER funds) is gratefully acknowledged. R.E.L. is thankful to SOLARNET for funding the visit to the Instituto de Astrofísica de Andalucía (CSIC) under the Mobility Programme for Young Researchers. The Swedish 1-m Solar Telescope is operated by the Institute for Solar Physics of Stockholm University in the Spanish Observatorio del Roque de los Muchachos of the Instituto de Astrofísica de Canarias. We thank the referee for the useful suggestions and comments. This research has made use of NASA's Astrophysical Data System.

\section{References}

Asai, A., Ishii, T. T., \& Kurokawa, H. 2001, ApJ, 555, L65

Berger, T. E., \& Berdyugina, S. V. 2003, ApJ, 589, L117

Bourdin, P.-A., Bingert, S., \& Peter, H. 2013, A\&A, 555, A123

Cabrera Solana, D., Bellot Rubio, L. R., \& del Toro Iniesta, J. C. 2005, A\&A, 439, 687

Cauzzi, G., Reardon, K. P., Uitenbroek, H., et al. 2008, A\&A, 480, 515

Cheung, M. C. M., Rempel, M., Title, A. M., \& Schüssler, M. 2010, ApJ, 720, 233

Choudhuri, A. R. 1986, ApJ, 302, 809

de la Cruz Rodríguez, J., De Pontieu, B., Carlsson, M., \& Rouppe van der Voort, L. H. M. 2013, ApJ, 764, L11

de la Cruz Rodríguez, J., Hansteen, V., Bellot-Rubio, L., \& Ortiz, A. 2015a, ApJ, 810,145

de la Cruz Rodríguez, J., Löfdahl, M. G., Sütterlin, P., Hillberg, T., \& Rouppe van der Voort, L. 2015b, A\&A, 573, A40

Drews, A. 2014, Master's Thesis, Institute of Theoretical Astrophysics, University of Oslo

Evershed, J. 1909, MNRAS, 69, 454

Fontenla, J. M., Avrett, E. H., \& Loeser, R. 1993, ApJ, 406, 319

Garcia de La Rosa, J. I. 1987, Sol. Phys., 112, 49

Giordano, S., Berrilli, F., Del Moro, D., \& Penza, V. 2008, A\&A, 489, 747

Guo, J., Liu, Y., Zhang, H., et al. 2010, ApJ, 711, 1057

Hirzberger, J., Bonet, J. A., Sobotka, M., Vázquez, M., \& Hanslmeier, A. 2002, A\&A, 383, 275

Illing, R. M. E., Landman, D. A., \& Mickey, D. L. 1975, A\&A, 41, 183
Jurčák, J., Martínez Pillet, V., \& Sobotka, M. 2006, A\&A, 453, 1079

Katsukawa, Y., Yokoyama, T., Berger, T. E., et al. 2007, PASJ, 59, 577

Leenaarts, J., Carlsson, M., Hansteen, V., \& Rouppe van der Voort, L. 2009, ApJ, 694, L128

Leka, K. D. 1997, ApJ, 484, 900

Lites, B. W., Scharmer, G. B., Berger, T. E., \& Title, A. M. 2004, Sol. Phys., 221,65

Liu, S. 2012, PASA, 29, 193

Louis, R. E., Bayanna, A. R., Mathew, S. K., \& Venkatakrishnan, P. 2008, Sol. Phys., 252, 43

Louis, R. E., Bellot Rubio, L. R., Mathew, S. K., \& Venkatakrishnan, P. 2009, ApJ, 704, L29

Louis, R. E., Ravindra, B., Mathew, S. K., et al. 2012, ApJ, 755, 16

Louis, R. E., Beck, C., \& Ichimoto, K. 2014, A\&A, 567, A96

Martínez González, M. J., \& Bellot Rubio, L. R. 2009, ApJ, 700, 1391

Matthews, S., Baker, D., \& Domínguez, S. V. 2012, in ASP Conf. Ser., 454, eds. T. Sekii, T. Watanabe, \& T. Sakurai, 209

Muller, R. 1979, Sol. Phys., 61, 297

Ortiz, A., Bellot Rubio, L. R., Hansteen, V. H., de la Cruz Rodríguez, J., \& Rouppe van der Voort, L. 2014, ApJ, 781, 126

Parker, E. N. 1979, ApJ, 234, 333

Reardon, K., Tritschler, A., \& Katsukawa, Y. 2013, ApJ, 779, 143

Rezaei, R., Bello González, N., \& Schlichenmaier, R. 2012, A\&A, 537, A19

Rimmele, T. 2008, ApJ, 672, 684

Rimmele, T., \& Marino, J. 2006, ApJ, 646, 593

Rimmele, T. R. 2004, ApJ, 604, 906

Rouppe van der Voort, L., Bellot Rubio, L. R., \& Ortiz, A. 2010, ApJ, 718, L78

Ruiz Cobo, B., \& del Toro Iniesta, J. C. 1992, ApJ, 398, 375

Scharmer, G. B., Bjelksjo, K., Korhonen, T. K., Lindberg, B., \& Petterson, B. 2003a, in SPIE Conf. Ser., 4853, 341

Scharmer, G. B., Dettori, P. M., Lofdahl, M. G., \& Shand, M. 2003b, in SPIE Conf. Ser., 4853, 370

Scharmer, G. B., Narayan, G., Hillberg, T., et al. 2008, ApJ, 689, L69

Schleicher, H., Balthasar, H., \& Wöhl, H. 2003, Sol. Phys., 215, 261

Schüssler, M., \& Vögler, A. 2006, ApJ, 641, L73

Selbing, J. 2010, ArXiv e-prints [arXiv: 1010.4142]

Shimizu, T. 2011, ApJ, 738, 83

Shimizu, T., Katsukawa, Y., Kubo, M., et al. 2009, ApJ, 696, L66

Sobotka, M., Bonet, J. A., \& Vazquez, M. 1994, ApJ, 426, 404

Sobotka, M., Švanda, M., Jurčák, J., et al. 2013, A\&A, 560, A84

Socas-Navarro, H., de la Cruz Rodríguez, J., Asensio Ramos, A., Trujillo Bueno, J., \& Ruiz Cobo, B. 2015, A\&A, 577, A7

Solanki, S. K., Lagg, A., Woch, J., Krupp, N., \& Collados, M. 2003, Nature, 425 , 692

Spruit, H. C., \& Scharmer, G. B. 2006, A\&A, 447, 343

van Noort, M., Rouppe van der Voort, L., \& Löfdahl, M. G. 2005, Sol. Phys., 228,191

Watanabe, H., Bellot Rubio, L. R., de la Cruz Rodríguez, J., \& Rouppe van der Voort, L. 2012, ApJ, 757, 49 